1

2

3

\title{
Modelling of storage tanks with immersed heat exchangers
}

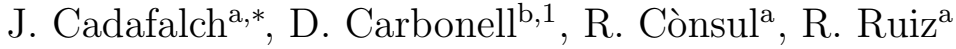 \\ ${ }^{a}$ Universitat Politècnica de Catalunya BarcelonaTech (UPC), Green Technologies \\ Resarch Group (GreenTech), Terrassa, Spain. \\ ${ }^{b}$ RDmes Technologies S.L., Ctra. Nac. 150, km 14.5, Institut Politècnic, 08227
}

Terrassa, Spain.

\begin{abstract}
A model of a storage tank with an immersed serpentine heat exchanger is described and validated against experimental data available from the literature. The tank is modelled one dimensionally using the multi-node approach corrected by an energy conservative reversion elimination algorithm to prevent inverse gradient solutions to occur. A one dimensional model in the flow direction is also used for the serpentine based on control volume techniques. The serpentine is discretized in equal sized control volumes and the energy equation is solved in each of them. The energy exchanged between the serpentine and the tank is then introduced as an internal heat source of the tank multi-node. With this model the behaviour of tanks with internal serpentines can be predicted minimizing tuning parameters to be derived from previous experimental analysis of the tank. Additionally, by an appropriate formulation of the governing equations in the serpentine control volumes, it is possible to handle complex internal fluid phenomena as coupling of the tank within a thermosyphone cycle or two phase flow.
\end{abstract}

Keywords:

Storage tanks, Modelling, Serpentine, Immersed heat exchanger

\footnotetext{
${ }^{*}$ Corresponding author

Email address: jcadafalch@mmt.upc.edu (J. Cadafalch)

${ }^{1}$ Present address: SPF Institut für Solartechnik, Hochschule für Technik (HSR), CH8640 Rapperswil, Switzerland.
} 


\section{Introduction}

In most solar thermal systems some kind of heat exchanger is used to separate the thermal fluid circulating through the collector field from the thermal fluid inside the tank. For medium or small size systems, with a store volume below around $2000 \mathrm{l}$, the heat exchanger is normally integrated within the tank. Mantle tanks or storages with immersed heat exchangers are the two main devices used for this purpose.

Mantle tanks, also known as jacket or annular heat exchangers, are the simplest and cheapest means of obtaining high thermal effectiveness while promoting stratification. However, as discussed by Furbo (1993) and Shah (2000), the use of mantle tanks is limited to volumes below 800-1000 l, because above these volumes heat transfer area reduces considerably. In tanks with immersed heat exchangers this problem is overcome. Additionally, the tank to ambient heat loss coefficient is also reduced.

Three and two dimensional models for storage tanks can be used to investigate specific phenomena. For example, the thermal stratification of cylindrical horizontal tanks has recently been analysed by Savicki et al. (2011); and Yan Su et al. (2008) studied the transient heat transfer characteristics of immersed serpentine heat exchangers. From these studies, very detailed information can be obtained. However, the computational demand is high, and the model must be run by specialists. As a consequence, they are not suitable for long term studies under operation conditions and cannot be used in market-oriented solar thermal systems engineering. Therefore, onedimensional tank approaches are still normally used for long term studies as those recently presented by Campos Celador et al. (2011), Young-Deuk Kim et al. (2012), Banister et al. (2014) or Shuhong Li et al. (2014), because they are able to offer an optimal compromise between accuracy and computational effort.

A widely used one-dimensional model for storage tanks is the multi-node model developed by Kleinbach et al. (1993). The tank is divided in different nodes from the bottom to the top, and the energy and mass conservation equations are solved at each of them over the time. This results into a one dimensional transient resolution of the tank.

An extension of the multi-node model was proposed by Newton et al. (1995) to include an immersed heat exchanger. This model assumes a constant heat transfer coefficient between the heat exchanger and storage fluid that must be introduced as an input value. 
A multi-port store model able to include four immersed heat exchangers was developed by Druck et al. (1994). The heat transfer coefficient must also be introduced by the user for specific conditions. Therefore, experimental data should be used to estimate this parameter, what is known as model tuning process. Time dependence of the heat transfer coefficient is then directly estimated by the model. This model is widespread in solar thermal system testing. However, the experimental data required for the tuning process is not always available for commercial stores.

Additionally, the multi-node model for storage tanks must also include a reversion elimination algorithm, see Mather et al. (2002). Basically, this algorithm forces that fluid temperatures of the different layers increase with height. When the storage tank is heated in positions different from the top, a positive density vertical gradient provoke a fluid instability, which in turns causes a mixing process due to natural convection. This process ends up with a uniformly mixed temperature in the upper layers. The multinode model is not able to directly solve the instantaneous highly convective phenomena that takes places. The reversion elimination algorithm overcomes this problem. The work presented by Newton et al. (1995) describes this algorithm. Basically the idea is to check if there exists a layer with higher temperature than its upper neighbour. If this occurs, the two layers are mixed. The problem here relies in how the layers are mixed. From the author's knowledge, a volumetric mixing process is chosen for most of the models, see for example the models of Newton et al. (1995) and Druck et al. (1994).

In this paper, a model for storage tanks with immersed serpentine heat exchangers is presented. The model does not need a previous tuning process for the serpentine, and includes an energy conservative reversion elimination mechanism. The serpentine is discretized in different control volumes in the fluid flow direction. The energy equation is then solved for each of them. The convective heat transfer coefficients in the inner fluid and from the serpentine walls to the store, are calculated from empirical correlations. With this approach, if an appropriate governing equations formulation is used inside the serpentine heat exchanger, the model can also be extended to more complex heat transfer analysis, as coupling of the serpentine within a thermosyphone loop, or use of phase change fluid in the serpentine, for example when a heat pump condenser is integrated in the storage tank.

The mathematical formulation and numerical resolution of the model is explained in the following section. Afterwards, a comparison of numerical 
results with experimental data available in the literature for two different test cases are presented in order to assess credibility to the model.

\section{Mathematical formulation}

\subsection{Modified multi-node}

The multi-node model for stratified storage tanks as described in Kleinbach et al. (1993) is used coupled with an immersed heat exchanger model.

The tank is divided into $N$ equal sized control volumes in the vertical direction called nodes. They are numbered from the bottom to the top, being $n=1$ the node at the bottom, and $n=N$ the node at the top of the tank. A single node assumes fully mixed tank, and increased number of nodes results into a more stratified tank.

The energy and mass conservation law is then solved at each node.

For simplicity, the formulation presented hereafter addresses a tank with a single immersed heat exchanger connected to a collector field loop, and a single draw-off loop.

The energy equation at a generic node $n$ according to the multi-node model reads as follows:

$$
\dot{Q}_{n}^{a c u m}+\dot{Q}_{n}^{\text {loss }}+\dot{Q}_{n}^{\text {mix }}=\dot{Q}_{n}^{\text {col }}-\dot{Q}_{n}^{\text {load }}
$$

The term $\dot{Q}_{n}^{a c u m}$ is the accumulated heat at the node in W. Using a first order scheme for the time derivatives, and a time increment of $\Delta \mathrm{t}$, this term is evaluated as a function of the temperature of the node at the current time step $T_{n}$ and the temperature of the node at the previous time step $T_{n}^{o}$ as:

$$
\dot{Q}_{n}^{a c u m}=\rho_{n} c_{p_{n}} V_{n} \frac{T_{n}-T_{n}^{o}}{\Delta t}
$$

where $c_{p_{n}}$ and $\rho_{n}$ are the storage fluid heat capacity and density, and $V_{n}$ the volume of the node.

The heat losses to ambient in Eq. 1 is represented as $\dot{Q}_{n}^{\text {loss }}$ and can be computed in terms of the ambient temperature $T^{a}$ and the node to ambient overall heat transfer coefficient $U A_{n}^{\text {loss }}$ :

$$
\dot{Q}_{n}^{\text {loss }}=U A_{n}^{\text {loss }}\left(T_{n}-T^{a}\right)
$$

The node to ambient heat transfer coefficient $U A_{n}^{\text {loss }}$ can be approximated multiplying the overall tank heat loss coefficient, $U A^{\text {tnk }}$, by the fraction of the tank external area that belongs to the analysed node: 
where $S_{n}^{a}$ is the surface contact area between the node $n$ and the ambient. Mixing effects between neighbouring nodes is considered by means of $\dot{Q}_{n}^{m i x}$. This term makes use of an equivalent conductivity coefficient $\Lambda$ that represents the level of mixing of the tank in stand by conditions (when there is not energy exchange with the collector and load loop). The $\Lambda$ coefficient accounts for thermal conductivity of the storage fluid, and for internal free convective phenomena that may occur due to the presence of thermal bridges in the walls or internal elements. Accordingly, the mixing term, is then calculated as a function of the nodes temperatures as follows:

$$
\dot{Q}_{n}^{m i x}=\frac{\Lambda}{\delta_{n+1}} S_{n}^{n+1}\left(T_{n}-T_{n+1}\right)+\frac{\Lambda}{\delta_{n-1}} S_{n}^{n-1}\left(T_{n}-T_{n-1}\right)
$$

where $T_{n+1}$ and $T_{n-1}$ are the top and bottom neighbour nodes, $\delta_{n+1}$ and $\delta_{n-1}$ the distance between the centre of the node and the center of the neighbour nodes, and $S_{n}^{n+1}$ and $S_{n}^{n-1}$ the node surface in contact with the neighbouring nodes. In case of vertical cylindrical tank, these two surfaces are identical. In other tank geometries such as horizontal tanks, these two surfaces may differ.

The two terms on the right in the energy balance as represented in Eq. 1 , are the energy introduced at the node from the collector loop $\dot{Q}_{n}^{c o l}$ and the energy removed to the load loop $\dot{Q}_{n}^{\text {load }}$. The energy removed by the load loop, is calculated from an enthalpy balance using an upwind scheme for the temperatures. Accordingly, it is evaluated as follows:

$$
\dot{Q}_{n}^{\text {load }}=\dot{m}^{\text {load }} c_{p_{n}}\left(T_{n}-T_{n}^{\text {load }}\right)
$$

where $\dot{m}^{\text {load }}$ is the instantaneous load mass flow rate, and $T_{n}^{\text {load }}$ the temperature of the fluid entering the node. In the nodes where the loop inlet is placed, this temperature takes the value of the inlet fluid flow temperature. In other nodes, it takes the value of the lower node $T_{n-1}$.

As in the standard multi-node model direct fluid exchange in the collector loop is assumed, the term $\dot{Q}_{n}^{c o l}$ in Eq. 1 is calculated using a similar procedure to that used for the load term $\dot{Q}_{n}^{\text {load }}$. To account for the effect of the immersed heat exchanger in the collector loop, $\dot{Q}_{n}^{c o l}$ is calculated from the immersed heat exchanger model described in subsection 2.2. This term is then renamed as: 
By applying the formulation given above, a set of algebraic equations (one for each node) is obtained being the temperature of the nodes at the current time step the unknowns. For an intermediate node the algebraic equation reads:

$$
A_{n}^{o} T_{n}^{o}+A_{n} T_{n}+A_{n+1} T_{n+1}+A_{n-1} T_{n-1}+\left(B+\dot{Q}_{n}^{h x}\right)=0
$$

As the algebraic coefficients $\mathrm{A}$ and B and the term $\dot{Q}_{n}^{h x}$ may depend on the nodes temperatures, the algebraic equation set is not linear. A iterative solver based on tridiagonal matrix algorithm is used to solve the resulting equations system, Patankar (1980).

\subsection{Immersed heat exchanger}

The serpentine immersed heat exchanger is evaluated with a step-bystep model as describer by Carbonell et al. (2013). The temperature of the fluid inside the serpentine is calculated by means of a one-dimensional analysis in the fluid flow direction applying a control volume discretization technique. The serpentine is discretized in $\mathrm{M}$ equal sized control volumes and the energy equation is solved at each of them, see Fig. 1. For convenience, the fluid temperatures are evaluated at the surfaces of the control volumes in the flow direction, and the temperature of the control volume is calculated from an averaging of the two corresponding temperatures. The calculated temperatures are represented by $T_{m}$ being $T_{1}$ and $T_{M+1}$ the temperature of the fluid at the inlet and outlet of the heat exchanger respectively. The average temperature of the control volume $m$, is then represented by $\overline{T_{m}}$, and is calculated as the average of $T_{m}$ and $T_{m+1}$. Accordingly, the energy equation at a control volume $m$ using a first order scheme for the time derivatives, and neglecting the heat axial conduction and the energy accumulation at the pipe walls, reads as follows:

$$
\rho c_{p} V \frac{\overline{T_{m}}-\overline{T_{m}^{0}}}{\Delta t}+\dot{m} c_{p}\left(T_{m+1}-T_{m}\right)+U A_{m}^{h x}\left(\overline{T_{m}}-T_{m_{e x t}}\right)=0
$$

where the superscript o in $\overline{T_{m}^{o}}$ refers to the value at the previous computed time step, $\rho$ and $c_{p}$ are the density and specific heat of the inner fluid, $\dot{m}$ is the inner mass flow rate, $\mathrm{V}$ the volume of fluid contained in the control volume, $U A_{m}^{h x}$ is the heat transfer coefficient from the inner fluid to the external fluid 
and $T_{m_{e x t}}$ is the temperature of the external fluid, i.e. the temperature of the tank node $n$ in which the serpentine control volume $m$ is placed.

The $M$ algebraic equations resulting from the discretized energy conservation law, Eq. 9, are solved following a step by step procedure (from the inlet to the outlet). The model needs no iterations if physical properties and $U A_{m}$ are calculated from the inlet fluid temperature $T_{m}$ for each control volume. The heat transferred from the serpentine to the tank node $n, \dot{Q}_{n}^{h x}$, is finally calculated from the summation of the heat loss term of all serpentine control volumes $C V s$ placed inside the tank node $n$ as follows:

$$
\dot{Q}_{n}^{h x}=\sum^{C V s} U A_{m}^{h x}\left(\overline{T_{m}}-T_{n}\right)
$$

\subsubsection{Heat transfer coefficient $U A_{m}^{h x}$}

The heat transfer coefficient from the serpentine internal fluid to the tank node fluid, is calculated according to the standard formulation of heat transfer through a cylindrical wall:

$$
U A_{m}^{h x}=\left[\frac{D_{\text {ext }}}{h_{\text {int }} D_{\text {int }}}+\frac{1}{\kappa^{\text {wall }}}+\frac{1}{h_{m_{\text {ext }}}}\right]^{-1} A_{m_{\text {ext }}}
$$

where $D_{\text {ext }}$ and $A_{m_{e x t}}$ are the external diameter and area of the pipe, $D_{\text {int }}$ means the internal diameter of the pipe, $\kappa^{\text {wall }}$ is the conductance of the pipe wall, and $h_{m_{e x t}}$ and $h_{i n t}$ the external and internal convective heat transfer coefficient of the pipe control volume $m$.

The pipe wall conductance is calculated referred to the external pipe area, and depends on the wall conductivity $\lambda^{\text {wall }}$ and the external and internal pipe diameters according to the following equation:

$$
\kappa^{\text {wall }}=\frac{\lambda^{\text {wall }}}{\frac{D_{e x t}}{2} \ln \frac{D_{e x t}}{D_{\text {int }}}}
$$

The internal convective heat transfer coefficient $h_{\text {int }}$, is obtained from experimental correlations of the Nusselt number for forced convective heat transfer fluid flow through cylindrical tubes as reported in Wong (1977), as a function of the Reynold and Prandtl dimensionless numbers:

$$
\frac{h_{i n t} D_{i n t}}{\lambda_{i n t}}=N u_{i n t}=C \operatorname{Re}^{m} \operatorname{Pr}^{n} K
$$


where $\lambda_{\text {int }}$ is the thermal conductivity of the inner fluid, $D_{\text {int }}$ the inner tube diameter, and the coefficients $C, m, n$ and $K$ depend on the flow regime, and the tube length, see Table 1.

The external convective heat transfer coefficient at the serpentine control volume $m h_{m_{e x t}}$ is calculated according to the expression of combined free and forced convection around immersed bodies described by Churchill (2002). The external Nusselt number at the control volume $m N u_{m_{e x t}}$ is calculated as the average of two Nusselts labelled with the super indexes + and -, which are calculated from a function combining the free (natural) convective Nusselt number $N u_{m}^{N}$ and the forced convective Nusselt number $N u_{m}^{F}$. The formulation is able to handle both positive (heating) and negative (cooling) heat transfer from the serpentine to the tank. Accordingly, $N u_{m_{\text {ext }}}$ is calculated with the following equation:

$$
\frac{h_{m_{e x t}} \pi D_{e x t}}{\lambda}=N u_{m_{e x t}}=\frac{N u_{m}^{+}+N u_{m}^{-}}{2}
$$

where $\mathrm{Nu}^{+}$and $\mathrm{Nu}^{-}$are obtained from the functions of the natural and forced convective Nusselt numbers:

$$
\begin{aligned}
& N u_{m}^{-}=1+\left|\left(N u_{m}^{F}\right)^{4}-\left(N u_{m}^{N}\right)^{4}\right|^{\frac{1}{4}} \\
& N u_{m}^{+}=1+\left|\left(N u_{m}^{F}\right)^{4}+\left(N u_{m}^{N}\right)^{4}\right|^{\frac{1}{4}}
\end{aligned}
$$

The forced Nusselt $N u_{m}^{F}$ is obtained from:

$$
N u_{m}^{F}=A_{F} \operatorname{Re}_{m}^{1 / 2} \operatorname{Pr}_{m}^{1 / 3}\left[1+\left(C_{F} / P r_{m}\right)^{2 / 3}\right]^{-1 / 4}
$$

where $R e_{m}$ and $\operatorname{Pr}_{m}$ are the Reynolds and Prandtl number respectively. The coefficients $A_{F}$ and $C_{F}$ are obtained as a function of the shape and boundary conditions. For a horizontal cylinder with uniform temperature (the closest approach) $A_{F}=1.08$ and $C_{F}=0.412$. The Reynolds number $R e_{m}$ is calculated using the tank fluid physical properties, the average velocity of the fluid circulating through the node in which the serpentine control volume $m$ is placed, and $\pi D_{\text {ext }}$ as characteristic length.

The natural Nusselt number at the serpentine control volume $m$ is obtained from a general expression proposed by Farrington et al. (1986):

$$
N u_{m}^{N}=C_{N}\left(G r_{m} P r_{m}\right)^{n_{N}}
$$


where the coefficients $C_{N}$ and $n_{N}$ are user defined. Farrington et al. (1986) suggested to fit this values with experimental data. However, as addressed by Newton et al. (1995) if this data is not available, the values of $C_{N}=0.5$ and $n_{N}=0.25$ can be used. The Prandtl and Grashof numbers $P r_{m}$ and $G r_{m}$ in Eq. 17 are calculated using the tank fluid physical properties of the corresponding node. The equation for $G r_{m}$ reads as follows:

$$
G r_{m}=\frac{g \beta \rho^{2}\left|\overline{T_{m}^{\text {wall }}}-T_{m_{\text {ext }}}\right|\left(\pi D_{\text {ext }}\right)^{3}}{\mu^{2}}
$$

where $\overline{T_{m}^{\text {wall }}}$ is the wall temperature of the serpentine control volume $m$ and $T_{m_{e x t}}$ the temperature of the corresponding tank node.

\subsection{Tuning parameters}

Beside geometrical parameters of the tank and the serpentine, the model requires some further information that can be obtained from experiments following standard procedures defined in EN 12977-3 (2012), or from a numerical evaluation of the tank with higher level numerical tools as for example is described in the work of Consul et al. (2004). Main parameters are the number of nodes to be used in the multi-node model $N$, the tank ambient heat loss coefficient $U A^{\text {tnk }}$ and the value of the coefficients to account for the effective conductive heat transfer between the nodes $\Lambda$.

To better predict the performance of the serpentine, the values $C_{N}$ and $n_{N}$ from Eq. 17 can also be adjusted from experimental data of the performance of the serpentine under different working conditions. However, such experimental data is usually not available for commercial storage tanks. In these cases, the standard values as discussed in Section 2.2.1 can be used, i.e. $C_{N}=0.5$ and $n_{N}=0.25$.

\subsection{Reversion elimination algorithm}

When a tank is heated up by means of a immersed heat exchanger placed at its bottom, negative density gradients may occur. As a consequence, a fluid motion is generated which in turns produces a fast mixing. The mixing process ends with a uniform temperature of the tank section above where the density gradient was present.

A detailed modelling of this phenomena can be carried out by direct resolution of the transient Navier-Stokes Equations for free-convection in 
order to take into account the different temporal and spacial characteristic scales.

The multi-node model approach, which is based in global energy and mass balances at the different nodes distributed vertically, may result into unrealistic temperature inversions when the tank is being heated up by a heat exchanger placed at its bottom. A so called reversion elimination algorithm for multi-node model was suggested by Newton et al. (1995) in order overcome this problem. Once mass and energy equation at each node are solved, a routine consisting of running through all the tank nodes from the top to the bottom is applied. If the temperature below the analysed node is higher, then the two nodes are mixed. If this temperature is still lower than the next node, the three are mixed. The procedure continues until a node with lower temperature is found. This algorithm is repeated until the criteria of $T_{n}>=T_{n-1}$ is fulfilled for all nodes. A key aspect of the method relies on how the temperature of the nodes involved in the process explained above are mixed.

A reversion elimination algorithm based on energy conservation is here presented.

Mixing process is achieved by setting the equivalent conductivity coefficient between the mixed nodes $\Lambda$ in Eq. 5, to infinity. This numerical trick results into zero temperature gradient solution between the mixed nodes.

For each simulated time step, the set of algebraic energy equations, see Eq. 8, is solved sequentially as times as necessary until temperature reversion is eliminated. The first computation is performed by using the $\Lambda$ physical values of the tank (according to the input data). After the first computation is finished, all $\Lambda$ values between those nodes with inverse temperatures are set to infinity, and the set of algebraic equations are solved for a second time. The $\Lambda$ coefficients between the nodes to be mixed is then kept to a value of infinity until the final time step solution is reached. As the solution of the second computation may also contain some temperature inversions, the procedure is carried out again. This procedure is repeated until no inverse temperatures are found. The total number of computations to achieve noneinverse temperature solutions ranges from 1 , in those cases where there is no any inversion, to $\mathrm{N}$ (being $\mathrm{N}$ the number of nodes used in the multi-node model), in those cases with maximum inverse temperature distribution. 


\section{Results}

Two different cases are used to validate the model. They both consist of a tank with an immersed serpentine heat exchanger placed at its bottom which introduces or removes energy from the tank following different patterns. Numerical results obtained with the methodology here described are validated by comparison to experimental data reported by Farrington et al. (1986) and Mather et al. (2002). Results are presented in figures reproducing the reporting criteria adopted by these authors to present their experimental data.

Computations here not reported have been carried out in order to investigate the sensitivity of the output analysed data on the discretization parameters $\mathrm{M}$ (number of control volumes in the serpentine) and $\Delta t$. The parameter M was varied from 5 to 1000, and the value of $\Delta t$ from 15 to 450 s. No significant differences were found in any case. Therefore, all numerical results here presented have been obtained with a $\Delta t$ of $450 \mathrm{~s}$ and with 5 control volumes in the serpentine in order to save computational effort.

The parameters that have been tuned to match the experiments are indicated in Table 2. Details of the tanks and heat exchangers geometry and discretization are shown in Fig. 2.

\subsection{Case 1: fully mixed tank}

A fully mixed tank is heated up by the serpentine with the inner fluid at a constant inlet temperature. Experimental results and details of the test procedure are reported by Farrington et al. (1986). The test starts with the storage tank with an initial temperature of $T_{i n i}^{t n k}=25^{\circ} \mathrm{C}$. Then, fluid at $T_{i n}^{h x}=70^{\circ} \mathrm{C}$ is circulated through the serpentine (inlet temperature), until the temperature difference between the inlet and outlet of the serpentine is below $0.1^{\circ} \mathrm{C}$. The test was preformed under different flow rates through the serpentine of 5,10 and $15 \mathrm{l} / \mathrm{min}$ respectively.

Comparison of numerical and experimental results are shown in Fig. 3. The serpentine effectiveness $\epsilon$, the overall heat transfer coefficient $U A^{h x}$ and the heat exchanger power $\dot{Q}^{h x}$ are analysed as a function of the logarithmic mean temperature difference $L M T D$ during the whole test period.

The instantaneous logarithmic mean temperature $L M T D$ is defined as:

$$
L M T D=\frac{T_{i n}^{h x}-T_{o u t}^{h x}}{\ln \frac{T_{i n}^{h x}-T^{t n k}}{T_{o u t}^{h x}-T^{t n k}}}
$$


where $T_{\text {in }}^{h x}$ and $T_{\text {out }}^{h x}$ refer to inlet and outlet temperature in the immersed heat exchanger and $T^{t n k}$ the temperature of the fully mixed tank.

The instantaneous effectiveness of the heat exchanger is defined by:

$$
\epsilon=\frac{T_{i n}^{h x}-T_{o u t}^{h x}}{T_{i n}^{h x}-T^{t n k}}
$$

and the overall heat transfer coefficient $U A^{h x}$ as:

$$
U A^{h x}=\frac{\dot{Q}^{h x}}{L M T D}
$$

where the $\dot{Q}^{h x}$ is the total heat exchanger heat flux in $[W]$.

Numerical results are in very good agreement with experimental data for all flow rates and over all the $L M T D$ range. Differences observed between experimental and numerical curves of $\epsilon, U A^{h x}$ and $\dot{Q}^{h x}$ for a specific $L M T D$ value are always kept below $5 \%$, while the statistical error of the experimental data as presented in Fig. 3 are also in the range of $\pm 5 \%$.

This case permits the validation of the immersed serpentine model under heating mode, however, no tank cooling process is tested. Furthermore, as a fully mixed tank is assumed by using a single node, the reversion elimination algorithm is neither validated. The validation of these two concepts is carried out with the test case 2 in the following subsection.

\subsection{Case 2: stratified tank with inverse temperature gradients}

The tank energy is varied by setting the inlet serpentine temperature in a series of four pulses so as the serpentine acts both heating and cooling the tank. A high level of stratification is assumed. As inverse temperature gradients occur, the reversion elimination algorithm is here necessary. This case is extracted from the work reported by Mather et al. (2002). In this report experimental an numerical results analysing the performance of single and multi-tanks with internal serpentine heat exchangers are presented. Further details on the tank technical data and testing conditions can be found there.

Values of the multi-node tank model parameters $\mathrm{N}, \Lambda$ and $U A^{\text {tnk }}$, together with additional tuning parameters to account for the effect of the serpentine were also experimentally obtained and reported by Mather et al. (2002). The tank model here reported differs from the model adopted by Mather et al. (2002) on the use of the energy conservative reversion elimination algorithm 
and the detailed modelling of the serpentine. As a result, only the tuning parameters of the multi-node model, i.e. $\mathrm{N}, \Lambda$ and $U A^{\text {tnk }}$ are required. Results here presented make use of the multi-node model parameters determined by Mather et al. (2002), see Table 2.

The tank is modelled with $N=21$ nodes and using a value of the equivalent conductivity coefficient $\Lambda$ of 1.3 times the thermal conductivity of the tank fluid (water). The nodes are labelled from 1 to 21 starting at the bottom. The node 1 is just below the serpentine, and the serpentine is located inside node 2.

The test starts with the tank at $20^{\circ} \mathrm{C}$. Then, water is circulated through the serpentine varying the input temperature $T_{i n}^{h x}$ each two hours, taking the value of $40^{\circ} \mathrm{C}, 60^{\circ} \mathrm{C}, 35^{\circ} \mathrm{C}$ and $25^{\circ} \mathrm{C}$ sequentially. With this test, the serpentine actuates both introducing and removing energy from the tank, and additionally, inverse temperature gradients situations occur that are handled numerically by the reversion elimination algorithm.

Comparison of the experimental data of Mather et al. (2002) and the results from the numerical model are presented in Fig. 4. Shown are the evolution of the inlet and outlet serpentine temperatures during the 8 hours test period, and the evolution of a selection of the tank nodes temperatures during the test period.

Again, the numerical results match the experimental data very well for all step change pulses along the time. Some discrepancies are observed in node 1 during the tank heating process taking place within the 4 first hours of the experiment. A similar behaviour was observed in the model used by Mather et al. (2002). During this period, all nodes are heated from below except node 1 . Therefore, the reversion elimination algorithm is actuating resulting into a totally mixed tank from node 2 to node 21 . On the contrary, node 1 is heated from above, and the model assumes that heat transfer from node 2 to 1 is only due to diffusion. The experiments, however, showed how node 1 was also mixed with other nodes due to the penetration of the convective flows generated at node 2 . This aspect is not accounted by the model here described. As concluded by Mather et al. (2002), however, as node 1 only constitutes a $5 \%$ of the total tank volume, and this effect only occurs during partial periods of the total test, the accuracy of the computed output temperature of the serpentine is not significantly affected. Modifications in the tank multi-node to account this aspect could be included. However, it would introduce additional model complexity that according to the authors concern may not be justified. 
During the last 4 hours of the experiment, the serpentine inlet temperature is below the tank nodes temperature. The reversion elimination algorithm actuates mixing the nodes 1 and 2. Heat transfer between other nodes is dominated by diffusion. Numerical results show very good agreement with the experimental values.

\section{Conclusions}

A model for thermal storage tanks with internal serpentine heat exchanger has been developed and presented in this work. The model combines the use of the standard multi-node approach for storage tanks, with a reversion elimination algorithm based on energy conservation, and a one-dimensional control volume approach for the fluid flow through the serpentine.

The use of the energy conservative reversion elimination algorithm results into a simple and consistent formulation that avoids unrealistic inverse temperature gradient solutions in the multi-node, while preserving energy conservation in all tank nodes and, therefore, in the whole tank. According to author's experience, conservative models in systems simulations improve convergence and robustness of the results. Additionally, programming aspects as identification of bugs and code verification are also facilitated.

The one dimensional control volume based model for the serpentine minimizes the required input technical data of the serpentine. Only with the geometrical parameters diameter, length and wall thickness it is possible to accurately evaluate its performance. Furthermore, with an appropriate formulation of the heat transfer and fluid flow governing equations, it may be possible to handle more complex physical phenomena inside the serpentine, like coupling of the serpentine in a thermosyphone loop, or two phase fluid flow.

The model has been validated under two test cases reported in the literature. These tests involve diverse working conditions as heating and cooling process of the tank through the serpentine, and inverse temperature gradients. Input values used for the computation were standard input data for the multi-node model and geometric data for the serpentine, with no need of specific performance data of the heat exchanger nor specific tuning of additional parameters for the heat exchanger. 


\section{Nomenclature}

$A \quad$ area $\left(m^{2}\right)$; algebraic coefficient in Eq. 8

$A_{F} \quad$ fitting coefficient in Eq. 16

$B \quad$ algebraic coefficient in Eq. 8

C fitting coefficient in Eq. 13

$C_{F} \quad$ fitting coefficient in Eq. 16

$C_{N} \quad$ fitting coefficient in Eq. 17

$C V s$ control volumes

$c_{p} \quad$ specific heat at constant pressure $(\mathrm{J} / \mathrm{kgK})$

$D \quad$ diameter $(m)$

Exp experimental

Gr Grashof number

$G z \quad$ Graetz number

$h \quad$ heat transfer coefficient $\left(W / m^{2} K\right)$

$K \quad$ fitting coefficient in Eq. 13

$L \quad$ total serpentine length $(m)$

$L M T D$ logarithmic mean temperature difference $\left({ }^{\circ} C\right)$

$M \quad$ total number of control volumes used in the serpentine

$\dot{m} \quad$ mass flow rate $(\mathrm{kg} / \mathrm{s})$

$N \quad$ total number of nodes used in the multi-node model

$n_{N} \quad$ fitting coefficient in Eq. 17

Num numerical

$\mathrm{Nu} \quad$ Nusselt number

$\operatorname{Pr} \quad$ Prandtl number

$\dot{Q} \quad$ heat $(W)$

Re Reynolds number

$S \quad$ surface $\left(m^{2}\right)$

$T \quad$ temperature $\left({ }^{\circ} \mathrm{C}\right)$

$U A \quad$ overall heat transfer coefficient $(W / K)$

$V \quad$ volume $\left(m^{3}\right)$

$\beta \quad$ volumetric coefficient of thermal expansion $(1 / K)$

$\Delta t \quad$ time increment $(s)$

$\delta \quad$ distance $(m)$

$\epsilon \quad$ heat exchanger effectiveness

$\kappa \quad$ conductance $\left(W / m^{2} K\right)$

$\Lambda \quad$ equivalent conductivity coefficient $(W / m K)$

$\lambda \quad$ thermal conductivity $(W / m K)$

$\rho \quad$ density $\left(\mathrm{kg} / \mathrm{m}^{3}\right)$

$\mu \quad$ dynamic viscosity $(\mathrm{kg} / \mathrm{sm})$ 


\section{Subscripts}

ext external

in at inlet

ini initial conditions

int internal

$m \quad$ control volume index in the serpentine model

$n \quad$ node index in the tank multi-node model

out at outlet

\section{Superscripts}

a ambient

acum accumulated heat

col collector

$F \quad$ forced convection

$h x \quad$ heat exchanger

load referred to heat load

loss referred to heat loss

$m \quad$ fitting coefficient in Eq. 13

mix mixing

$N$ natural (free) convection

$n \quad$ fitting coefficient in Eq. 13

$o \quad$ value at the previous time step

tnk tank

wall referred to wall 


\section{References}

Banister, Carsen J., Wagar, William R. and Collins, Prof. Michael R., 2014. Validation of a single tank, multi-mode solar-assisted heat pump TRNSYS model. Energy Procedia 48 (2014), 499-504.

Campos Celador, A., Odriozola, M. and Sala, J.M., 2011. Implications of the modelling of stratified hot water storage tanks in the simulation of the CHP plants. Energy Conversion and Management, 52 (2011), 3018-3026.

Carbonell, D., Cadafalch, J. and Consul, R., 2013. Dynamic modelling of flat plate solar collectors. Solar Energy, 89, 100-112.

Churchill, S.W., 2002. Combined free and forced convection around immersed bodies. Heat Exchanger Design handbook, Hewitt G.F., sec. 2.5.9. Hemisphere Publishing Corporation.

Consul, R., Rodríguez, I., Pérez-Segarra, C.D., and Oliva, A., 2004. Virtual prototyping of storage tanks by means of three-dimensional cfd and heat transfer numerical simulations. Solar Energy, 77, 179-191.

Drück, H., 1994. Weiterentwicklung und validierung des modells für solare Warmwasserspeicher für das simulationsprogramm TRANSYS. Diplomarbeit ITW, Universität Stuttgart.

EN 12977-3, 2012. European Standard. Thermal solar systems and components - Custom built systems - Part 3: Performance test methods for solar water heater stores European Committee for Standardisation.

Farrington, R.B. and Bingham, C.E., 1986. Analysis of Immersed heat exchangers, Solar Energy Research Institute, Golden, CO.

Furbo, S., 2003. Optimum design of small DHW low flow solar heating systems. In Proceedings, ISES solar World Congress, Budapest, Hungary.

Kleinbach, E.M., Beckman, W.A. and Klein, S.A., 1993. Performance study of one-dimensional models for stratified thermal storage tanks. Solar Energy, 50 (2), 155-166.

Mather, D.W., Hollands, K.G.T and Wright J.L., 2002. Single and multi-tank energy storage for solar heating systems: fundamentals. Solar Energy, 73 (1), 3-13. 
Newton, B.J., Schmid M., Michell J.W. and Beckman, W.A., 1995. Storage tank models. Proceedings of ASME/JSME International Solar Energy Conference, March 19-24, 1995. Maui, Hawaii. American Society of Mechanical Engineers, New York, 2 , 1111-1116.

Patankar, S.V., 1980. Numerical heat transfer and fluid flow. Hemisphere.

Savicki, Darci L., Vilemo, Horcio A. and Krenzinger, A., 2011. Threedimensional analysis and investigation of the thermal and hydrodynamic behaviours of cylindrical storage tanks. Renewable Energy 36 (2011), 13641373 .

Shah, L.J., 2000. Heat transfer correlations for vertical mantle heat exchangers. Solar Enery, 96, 157-171.

Shuhong Li, Yongxin Zhang, Kai Zhang, Xianliang Li, Yang Li and Xiaosong Zhang, 2014. Study on performance of storage tanks in solar water heater system in charge and discharge progress. Energy Procedia 48 (2014), 384393.

Wong, H.Y., 1977. Handbook of essential formulae and data on heat transfer for engineers. Longman Group Limited London.

Yan Su and Davidson, Jane H., 2008. Discharge of thermal storage tanks via immersed baffled heat exchangers: numercial model of flow and temperature fields. J. Sol. Energy Eng., 130 (2), doi:10.1115/1.2856012

Young-Deuk Kim, Kyaw Thu, Hitasha Kaur Bhatia, Charanjit Singh Bhatia and Kim Choon Ng, 2012. Thermal analysis and performance optimization of a solar hot water plant with economic evalutaion. Solar Energy, 86 (5), 1378-1395. 
Table 1: Forced convective heat transfer of fluid flow through a cylindrical tube. Experimental fitting parameters in terms of the operating conditions, Wong (1977). The Graetz number $\mathrm{Gz}$ is defined as $\mathrm{Gz}=\left(\pi D_{\text {int }} / 4 L\right) \operatorname{Re} \operatorname{Pr}$, and $\mathrm{L}$ is the total serpentine length.

\begin{tabular}{lcccc}
\hline \hline Operating conditions & $\mathrm{C}$ & $\mathrm{m}$ & $\mathrm{n}$ & $\mathrm{K}$ \\
\hline $\begin{array}{l}\text { Laminar flow short tube } \\
\mathrm{Re}<=2000, \mathrm{Gz}>10\end{array}$ & 1.86 & $1 / 3$ & $1 / 3$ & $\left(D_{\text {int }} / L\right)^{1 / 3}$ \\
$\begin{array}{l}\text { Laminar flow long tube } \\
\mathrm{Re}<=2000, \mathrm{Gz}<10\end{array}$ & 3.66 & 0 & 0 & 1 \\
$\begin{array}{l}\text { Turbulent flow } \\
\operatorname{Re}>2000\end{array}$ & 0.027 & 0.8 & 0.33 & 1 \\
\hline \hline
\end{tabular}


Table 2: Values of the tuning parameters used in the test cases. *) Thermal conductivity of the tank fluid (water).

\begin{tabular}{cccccc}
\hline \hline Case & $\mathrm{N}$ & $\Lambda$ & $U A^{\text {tnk }}$ & $C_{N}$ & $n_{N}$ \\
& - & {$[\mathrm{W} / \mathrm{mK}]$} & {$[\mathrm{W} / \mathrm{K}]$} & - & - \\
\hline 1 & 1 & - & 1.600 & 0.5 & 0.25 \\
2 & 21 & $1.3 \lambda^{*}$ & 0.569 & 0.5 & 0.25 \\
\hline \hline
\end{tabular}




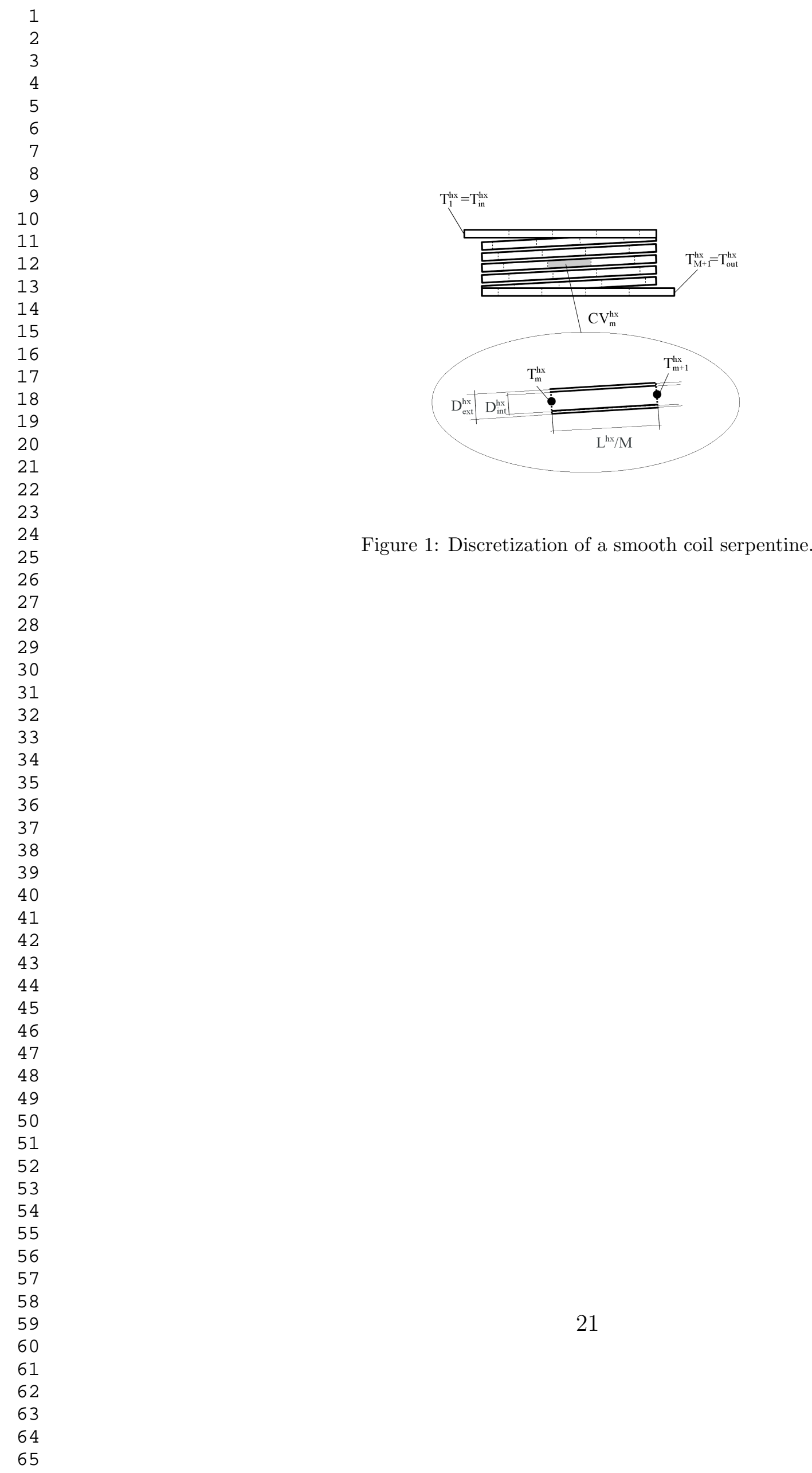




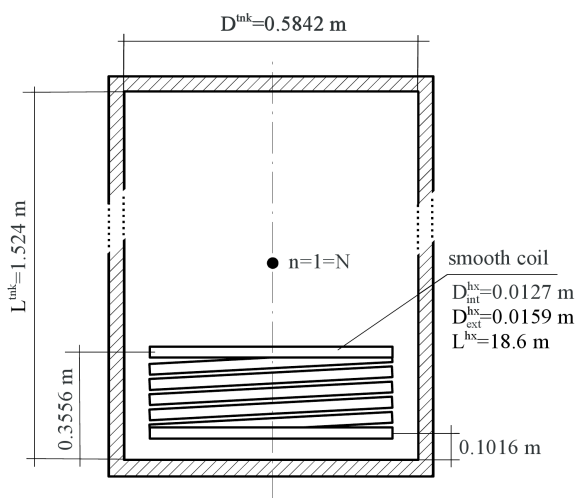

a)

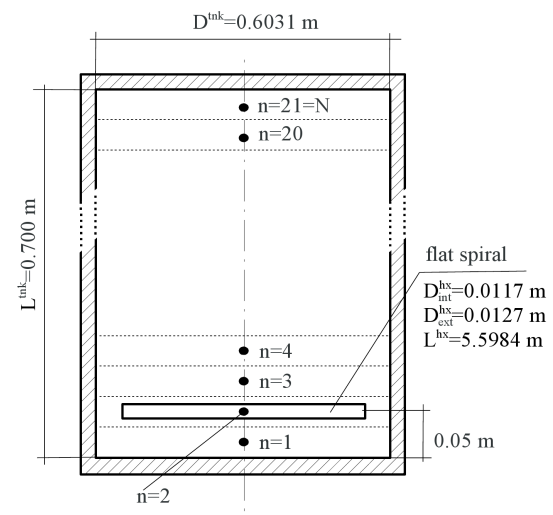

b)

Figure 2: Geometry and tank discretization of the case studies. a) Case 1: fully mixed tank. b) Case 2: stratified tank. 
Figure 3: Case 1: fully mixed tank. Immersed heat exchanger efficiency $\epsilon$, heat transfer coefficient $U A^{h x}$ and power $\dot{Q}^{h x}$ along the $L M T D$. Comparison between numerical results and experimental data from Farrington et al. (1986). 

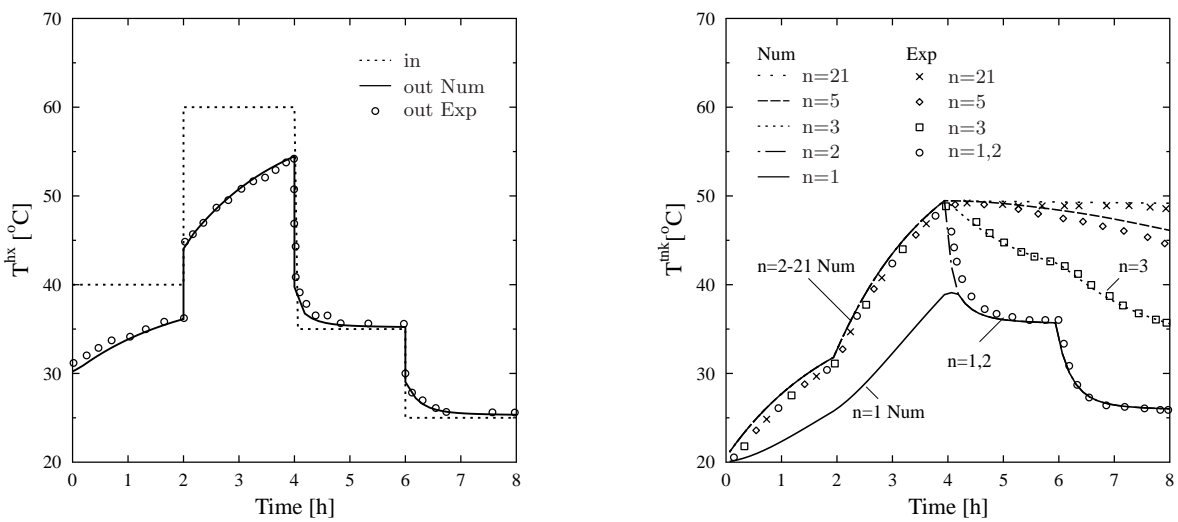

Figure 4: Case 2: stratified tank. Outlet fluid temperature of the immersed heat exchanger and tank temperature for different nodes along time. Comparison between numerical results and experimental data from Mather et al. (2002). 